\title{
High order approximation of degree nine and order eighteen
}

\section{Abedallah Rababah ${ }^{1 *}$ (DD}

"Correspondence: rababah@just.edu.jo

${ }^{1}$ Department of Mathematics and Statistics, Jordan University of Science and Technology, Irbid, Jordan

\begin{abstract}
In this paper, a method to approximate curves by polynomials of degree nine is presented. The resulting approximation has order eighteen. The method is applied to approximate a circular arc, and the error function is studied and characterized, and its extrema and zeros are derived.
\end{abstract}

Keywords: Bézier curves; High order approximation; Nonic polynomials

\section{Introduction}

Approximation methods for planar curves that sufficiently improve the standard approximation rates via Taylor's method are stated in $[10,11]$. The methods are based on the fact that the parametrization of a curve is not unique and can be suitably modified to improve the approximation order.

Given a planar curve

$$
C: t \rightarrow\left(\begin{array}{l}
f(t) \\
g(t)
\end{array}\right), \quad t \in \Re
$$

that is regular and smooth. In many applications, it is essential to approximate the curve $C$ by a polynomial curve of the form

$$
P: t \rightarrow\left(\begin{array}{c}
X_{m}(t) \\
Y_{m}(t)
\end{array}\right), \quad t \in \Re
$$

where $X_{m}(t)$ and $Y_{m}(t)$ are polynomials of degree $m$. The classical methods of approximation assure that the polynomial curve $P$ approximates the curve $C$ with order $m+1$. It is shown [10] that an improvement over the standard order $m+1$ is possible by creating new parameters depending on data of the curve. Without loss of generalization, it is assumed that $(f(0), g(0))=(0,0),\left(f^{\prime}(0), g^{\prime}(0)\right)=(1,0)$. In this case, for small $\mathrm{t}$, the curve $C$ can be parametrized in the form

$$
C: t \rightarrow\left(\begin{array}{c}
X_{m}(t) \\
\phi\left(X_{m}(t)\right)
\end{array}\right)
$$

(c) The Author(s) 2019. This article is distributed under the terms of the Creative Commons Attribution 4.0 International License (http://creativecommons.org/licenses/by/4.0/), which permits unrestricted use, distribution, and reproduction in any medium, provided you give appropriate credit to the original author(s) and the source, provide a link to the Creative Commons license, and indicate if changes were made. 
for a suitable $\phi\left(X_{m}(t)\right)$. The polynomial curve $P$ approximates the curve $C$ with the order $\alpha$ iff

$$
\phi\left(X_{m}(t)\right)-Y_{m}(t)=O\left(t^{\alpha}\right)
$$

The Hermite type conditions become

$$
\begin{aligned}
& \left.\frac{d^{j}}{d t^{j}}\left\{\phi\left(X_{m}(t)\right)-Y_{m}(t)\right\}\right|_{t=0}=0, \quad j=0,1, \ldots, \alpha_{1}-1, \\
& \left.\frac{d^{j}}{d t^{j}}\left\{\phi\left(X_{m}(t)\right)-Y_{m}(t)\right\}\right|_{t=1}=0, \quad j=0,1, \ldots, \alpha_{2}-1, \\
& X_{m}(0)=0, \quad X_{m}(1)=1, \quad \alpha_{1}+\alpha_{2}=\alpha .
\end{aligned}
$$

Setting $X_{m}^{\prime}(0)=1$, then the problem is characterized by the $2 m$ free parameters. The number of equations is compared with the number of parameters to get the order of approximation of $2 m$; this is justified in [10]. This is the Hermite-type interpolation to represent a curve. In this paper, the issue is to find the polynomial of best uniform approximation. The classical case of approximating using a polynomial of degree $m$ that has best uniform approximation was studied by Chebyshev and Borel [9]. They showed that such a polynomial exists and is unique, and its error equioscillates $m+2$ times; but so far there has been no method to find the approximating polynomial. The polynomial of best approximation of degree nine is found in this paper; it has nineteen equioscillations rather than eleven equioscillations theoretically guaranteed by the theorems of Chebyshev and Borel but cannot be found. The cases of $n=2,3,4$ are considered in [12-14]; i.e., the nonic piecewise approximation for planar curves $\alpha_{1}=\alpha_{2}=9$ is studied. The approximation order is raised to eighteen rather than ten.

In this paper, a curve that is most frequently used in applications, the circle, is considered. The polynomial of degree nine of best approximation that equioscillates 19 times rather than 11 times is found. This is a substantial improvement over the order of approximation.

Parametric forms of curves and surfaces are computer-oriented representations that can be easily used for graphing and computations. We treat the circular arc $c: t \mapsto$ $(\cos (t), \sin (t)),-\theta \leq t \leq \theta$, see Fig. 1 , to be approximated by a polynomial curve with superior uniform approximation. To come to this consequence, the geometric symmetries of the circle are used to fairly choose the Bézier points in order to symbolize the nonic Bézier curve that has the highest approximation order of 18 and has "the best" features.

The circle $c$ is approximated in this paper using a nonic parametrically defined polynomial curve $p: t \mapsto(x(t), y(t)), 0 \leq t \leq 1$, where $x(t), y(t)$ are polynomials of degree nine that approximate $c$ with "minimum" error. Many researchers have tackled this issue using different degrees, norms, and methods, see $[1-4,6,8,13]$. The results of our method in this paper are optimal and cannot be improved because the error function is the Chebyshev polynomial of first kind of degree eighteen.

The error between $p$ and $c$ is measured by the Euclidean error function

$$
E(t):=\sqrt{x^{2}(t)+y^{2}(t)}-1 .
$$


Figure 1 A circular arc

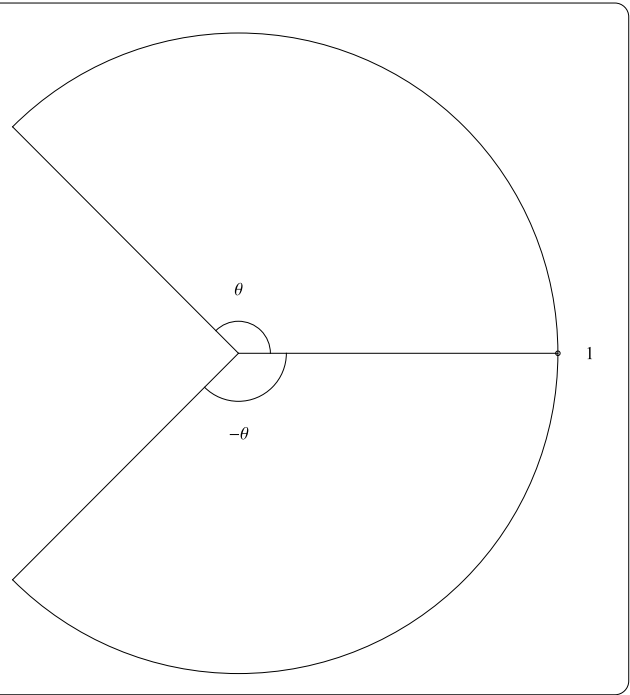

It is replaced by the following alternative error function:

$$
e(t):=x^{2}(t)+y^{2}(t)-1
$$

Since $e(t)=0$ is the implicit equation of the unit circle; this implies that the $e(t)$ error function is a suitable measure to test if $x(t)$ and $y(t)$ satisfy this equation and to measure the error. Both error functions share the same roots and critical points, see Propositions I and II.

The issue considered in this paper is to locate a polynomial curve $p: t \mapsto(x(t), y(t))$, $0 \leq t \leq 1$, where $x(t), y(t)$ are of degree nine, that approximates $c$ under the requirements of the best uniform approximation of the form:

1. $p$ minimizes $\max _{t \in[0,1]}|e(t)|$,

2. $e(t)$ equioscillates 19 times over $[0,1]$ rather than 11 times [9],

3. $p(t)$ approximates $c(t)$ with order 18 rather than 10 .

These conditions are used to locate the Bézier points and to get the values of the parameters that are utilized to satisfy the geometric conditions of the circular arc [5,7]. Since the uniform error (for $e(t)$ ) equals $2^{-17}$ [9], so we allow the angle $\theta$ to be as large as possible in order to approximate the largest circular arc with this specified error. Thereafter, this angle $\theta$ has to be scaled by a factor that is also combined with a reduction in the uniform error; this is a proposal for further future investigation.

The Bézier form is used to write the curve $p(t)$. The Bézier curve $p(t)$ of degree nine is given by

$$
p(t)=\sum_{i=0}^{9} p_{i} B_{i}^{9}(t)=:\left(\begin{array}{l}
x(t) \\
y(t)
\end{array}\right), \quad 0 \leq t \leq 1 .
$$

The points $p_{0}, p_{1}, p_{2}, p_{3}, p_{4}, p_{5}, p_{6}, p_{7}, p_{8}$, and $p_{9}$ are called the Bézier points, and the nonic polynomials $B_{0}^{9}(t)=(1-t)^{9}, B_{1}^{9}(t)=9 t(1-t)^{8}, B_{2}^{9}(t)=36 t^{2}(1-t)^{7} 6, B_{3}^{9}(t)=84 t^{3}(1-$ $t)^{6}, B_{4}^{9}(t)=126 t^{4}(1-t)^{5}, B_{5}^{9}(t)=126 t^{5}(1-t)^{4}, B_{6}^{9}(t)=84 t^{6}(1-t)^{3}, B_{7}^{9}(t)=36 t^{7}(1-t)^{2}$, $B_{8}^{9}(t)=9 t^{8}(1-t)$, and $B_{9}^{9}(t)=t^{9}$ are the Bernstein polynomials. Our purpose is to represent 
the curve using nonic polynomials under the condition that the error has order eighteen. Consequently, it is not substantial for the errors to take place at the endpoints or elsewhere.

Our approach considers lessening the wrongdoing over all of the segment $[0,1]$. To explore the Bézier form approximation of a circular arc, a careful selection of locations of the Bézier points should be done. These locations are substantial to earn the convenient curve that redeems the approximation conditions. Based on the symmetry property of the circle, the right choice for the beginning control point $p_{0}$ should obey the following form: $p_{0}=\left(\alpha_{0} \cos (\theta), \beta_{0} \sin (\theta)\right)$, where values of $\alpha_{0}$ and $\beta_{0}$ could but should not be the same. Similarly, for symmetry reasoning, the valid option for the end control point $p_{9}$ is $p_{9}=\left(\alpha_{0} \cos (\theta),-\beta_{0} \sin (\theta)\right)$. Set $p_{1}=\left(a_{1}, b_{1}\right)$, then the point $p_{8}$ has to be selected to satisfy the form $p_{8}=\left(a_{1},-b_{1}\right)$. Set the point $p_{2}=\left(a_{2}, b_{2}\right)$, then the point $p_{7}$ has to be selected to satisfy the form $p_{7}=\left(a_{2},-b_{2}\right)$. Set the point $p_{3}=\left(a_{3}, b_{3}\right)$, then the point $p_{6}$ for symmetry purposes has to be selected to satisfy the form $p_{6}=\left(a_{3},-b_{3}\right)$. Set the point $p_{4}$ of the form $p_{4}=\left(a_{4}, b_{4}\right)$, then the point $p_{5}$ for symmetry purposes has to be selected to satisfy the form $p_{5}=\left(a_{4},-b_{4}\right)$. Using the substitutions $a_{0}=\alpha_{0} \cos (\theta), b_{0}=\beta_{0} \sin (\theta)$, then the convenient choices for the Bézier points have to be, see Fig. 2:

$$
\begin{array}{ll}
p_{0}=\left(\begin{array}{l}
a_{0} \\
b_{0}
\end{array}\right), \quad p_{1}=\left(\begin{array}{l}
a_{1} \\
b_{1}
\end{array}\right), \quad p_{2}=\left(\begin{array}{l}
a_{2} \\
b_{2}
\end{array}\right), \quad p_{3}=\left(\begin{array}{l}
a_{3} \\
b_{3}
\end{array}\right), \\
p_{4}=\left(\begin{array}{c}
a_{4} \\
b_{4}
\end{array}\right), \quad p_{5}=\left(\begin{array}{c}
a_{4} \\
-b_{4}
\end{array}\right), \quad p_{6}=\left(\begin{array}{c}
a_{3} \\
-b_{3}
\end{array}\right), \\
p_{7}=\left(\begin{array}{c}
a_{2} \\
-b_{2}
\end{array}\right), & p_{8}=\left(\begin{array}{c}
a_{1} \\
-b_{1}
\end{array}\right), \quad p_{9}=\left(\begin{array}{c}
a_{0} \\
-b_{0}
\end{array}\right) .
\end{array}
$$

It will be apparent that there are more than one solution; the consonant solution of best approximation begins in the second quadrant and ends in the fourth quadrant counter clockwise. Therefore, in order to have the Bézier curve $p$ begin in the second quadrant, go counter clockwise through fourth, third, first, second, and end in the fourth quadrant as the circular $\operatorname{arc} c$, the following stipulations should be satisfied:

$$
a_{0}, a_{1}, a_{2}, b_{1}, b_{2}, b_{3}<0, \quad a_{3}, a_{4}, b_{0}, b_{4}>0 .
$$

Figure 2 The half of the solution for $0 \leq t \leq \frac{1}{2}$

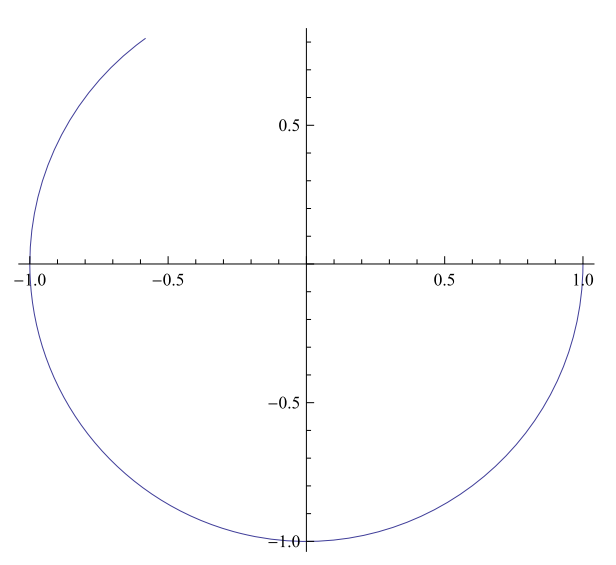


Employ the Bézier points in (4) in the Bézier curve $p(t)$ in (3) to obtain, for all $t \in[0,1]$,

$$
p(t)=\left(\begin{array}{l}
a_{0}\left(B_{0}^{9}(t)+B_{9}^{9}(t)\right)+a_{1}\left(B_{1}^{9}(t)+B_{8}^{9}(t)\right)+a_{2}\left(B_{2}^{9}(t)+B_{7}^{9}(t)\right)+a_{3}\left(B_{3}^{9}(t)+B_{6}^{9}(t)\right)+a_{4}\left(B_{4}^{9}(t)+B_{5}^{9}(t)\right) \\
b_{0}\left(B_{0}^{9}(t)-B_{9}^{9}(t)\right)+b_{1}\left(B_{1}^{9}(t)-B_{8}^{9}(t)\right)+b_{2}\left(B_{2}^{9}(t)-B_{7}^{9}(t)\right)+b_{3}\left(B_{3}^{9}(t)-B_{6}^{9}(t)\right)+b_{4}\left(B_{4}^{9}(t)-B_{5}^{9}(t)\right)
\end{array}\right) .
$$

The Bézier curve is settled by the ten parameters $a_{0}, a_{1}, a_{2}, a_{3}, a_{4}, b_{0}, b_{1}, b_{2}, b_{3}, b_{4}$ which are hired to get the best uniform approximation. We want to impose the conditions on the polynomial curve $p$; the polynomials $x(t)$ and $y(t)$ are substituted into $e(t)$. This leads to a polynomial of degree eighteen that is solved using a computer algebra system. The results are stated in the next section.

\section{Results}

In this section, the main results are stated. The values of $a_{0}, a_{1}, a_{2}, a_{3}, a_{4}, b_{0}, b_{1}, b_{2}$, $b_{3}, b_{4}$ that satisfy the conditions of the approximation problem are specified numerically, rounded fittingly, in the following theorem.

Theorem 1 The Bézier curve (8) together with the Bézier points in (9) and the values of the parameters $a_{0}, a_{1}, a_{2}, a_{3}, a_{4}, b_{0}, b_{1}, b_{2}, b_{3}, b_{4}$ given by

$$
\begin{aligned}
& a_{0}=-0.5828949409686828, \quad a_{1}=-1.8244399212283737, \\
& a_{2}=-0.9872644054741855, \quad a_{3}=2.8637303470555153, \\
& a_{4}=0.53960354850848, \quad b_{0}=0.8125522242832429, \\
& b_{1}=-0.07842428718981986, \quad b_{2}=-2.702352393721596, \\
& b_{3}=-1.6331336305047865, \quad b_{4}=1.7030404000205088
\end{aligned}
$$

achieves the following conditions: $p$ minimizes the uniform norm of the error function $\max _{t \in[0,1]}|e(t)|$, has eighteenth order of approximation, and the error function e $(t)$ equioscillates 19 times in $[0,1]$. The error functions fulfill, for all $t \in[0,1]$ :

$$
\begin{aligned}
& -\frac{1}{2^{17}} \leq e(t) \leq \frac{1}{2^{17}}, \quad-\frac{1}{2^{17}(2-\epsilon)} \leq E(t) \leq \frac{1}{2^{17}(2+\epsilon)}, \\
& \text { where } \epsilon=\max _{0 \leq t \leq 1}|E(t)| \approx 2^{-18} .
\end{aligned}
$$

The most important characteristics of the error functions are the roots and the extrema. These properties characterize the approximating nonic Bézier curve. The first characteristic concerns the roots of the error functions $e(t)$ and $E(t)$ that are specified in the following proposition.

Proposition I The roots of the error functions $e(t)$ and $E(t)$ are:

$$
\begin{array}{ll}
t_{1}=\frac{1}{2}\left(1+\cos \left(\frac{\pi}{36}\right)\right)=0.998097, & t_{2}=\frac{1}{2}\left(1+\cos \left(\frac{3 \pi}{36}\right)\right)=0.982963 \\
t_{3}=\frac{1}{2}\left(1+\cos \left(\frac{5 \pi}{36}\right)\right)=0.953154, & t_{4}=\frac{1}{2}\left(1+\cos \left(\frac{7 \pi}{36}\right)\right)=0.909576
\end{array}
$$




$$
\begin{aligned}
& t_{5}=\frac{1}{2}\left(1+\cos \left(\frac{9 \pi}{36}\right)\right)=0.853553, \quad t_{6}=\frac{1}{2}\left(1+\sin \left(\frac{7 \pi}{36}\right)\right)=0.786788, \\
& t_{7}=\frac{1}{2}\left(1+\sin \left(\frac{5 \pi}{36}\right)\right)=0.711309, \quad t_{8}=\frac{1}{2}\left(1+\sin \left(\frac{3 \pi}{36}\right)\right)=0.62941, \\
& t_{9}=\frac{1}{2}\left(1+\sin \left(\frac{\pi}{36}\right)\right)=0.543578, \quad t_{10}=\frac{1}{2}\left(1-\sin \left(\frac{\pi}{36}\right)\right)=0.456422, \\
& t_{11}=\frac{1}{2}\left(1-\sin \left(\frac{3 \pi}{36}\right)\right)=0.37059, \quad t_{12}=\frac{1}{2}\left(1-\sin \left(\frac{5 \pi}{36}\right)\right)=0.288691 \text {, } \\
& t_{13}=\frac{1}{2}\left(1-\sin \left(\frac{7 \pi}{36}\right)\right)=0.213212, \quad t_{14}=\frac{1}{2}\left(1-\cos \left(\frac{9 \pi}{36}\right)\right)=0.146447, \\
& t_{15}=\frac{1}{2}\left(1-\cos \left(\frac{7 \pi}{36}\right)\right)=0.090424, \quad t_{16}=\frac{1}{2}\left(1-\cos \left(\frac{5 \pi}{36}\right)\right)=0.0468461 \text {, } \\
& t_{17}=\frac{1}{2}\left(1-\cos \left(\frac{3 \pi}{36}\right)\right)=0.0170371, \quad t_{18}=\frac{1}{2}\left(1-\cos \left(\frac{\pi}{36}\right)\right)=0.00190265 \text {. }
\end{aligned}
$$

They also satisfy

$$
t_{i}+t_{j}=1 \quad \text { for } i+j=19
$$

The approximating nonic Bézier curve $p$ in Theorem 1 and the circular arc $c$ intersect at the points $p\left(t_{i}\right)=c\left(t_{i}\right), i=1,2, \ldots, 18$.

Regarding the extreme values, we have the following proposition.

Proposition II The extreme values of $e(t)$ and $E(t)$ occur at the parameters:

$$
\begin{aligned}
& \tilde{t}_{0}=1, \quad \tilde{t}_{1}=\frac{1}{2}\left(1+\cos \left(\frac{\pi}{18}\right)\right)=0.992404, \quad \tilde{t}_{2}=\frac{1}{2}\left(1+\cos \left(\frac{\pi}{9}\right)\right)=0.969846, \\
& \tilde{t}_{3}=\frac{1}{2}\left(1+\cos \left(\frac{\pi}{6}\right)\right)=0.933013, \quad \tilde{t}_{4}=\frac{1}{2}\left(1+\cos \left(\frac{2 \pi}{9}\right)\right)=0.883022, \\
& \tilde{t}_{5}=\frac{1}{2}\left(1+\cos \left(\frac{5 \pi}{18}\right)\right)=0.821394, \quad \tilde{t}_{6}=\frac{1}{2}\left(1+\cos \left(\frac{\pi}{3}\right)\right)=0.75, \\
& \tilde{t}_{7}=\frac{1}{2}\left(1+\cos \left(\frac{7 \pi}{18}\right)\right)=0.67101, \quad \tilde{t}_{8}=\frac{1}{2}\left(1+\cos \left(\frac{4 \pi}{9}\right)\right)=0.586824, \\
& \tilde{t}_{9}=\frac{1}{2}\left(1+\cos \left(\frac{\pi}{2}\right)\right)=0.5, \quad \tilde{t}_{10}=\frac{1}{2}\left(1-\cos \left(\frac{2 \pi}{9}\right)\right)=0.413176, \\
& \tilde{t}_{11}=\frac{1}{2}\left(1-\cos \left(\frac{7 \pi}{18}\right)\right)=0.32899, \quad \tilde{t}_{12}=\frac{1}{2}\left(1-\cos \left(\frac{\pi}{3}\right)\right)=0.25, \\
& \tilde{t}_{13}=\frac{1}{2}\left(1-\cos \left(\frac{5 \pi}{18}\right)\right)=0.178606, \quad \tilde{t}_{14}=\frac{1}{2}\left(1-\cos \left(\frac{2 \pi}{9}\right)\right)=0.116978, \\
& \tilde{t}_{15}=\frac{1}{2}\left(1-\cos \left(\frac{\pi}{6}\right)\right)=0.0669873, \quad \tilde{t}_{16}=\frac{1}{2}\left(1-\cos \left(\frac{\pi}{9}\right)\right)=0.0301537, \\
& \tilde{t}_{17}=\frac{1}{2}\left(1-\cos \left(\frac{\pi}{18}\right)\right)=0.00759612, \quad \tilde{t}_{18}=0 .
\end{aligned}
$$


These parameters satisfy the equality

$$
\tilde{t}_{i}+\tilde{t}_{j}=1 \quad \text { for } i+j=18
$$

The exact values of the extrema are given in the following proposition.

Proposition III The values of the error functions $e(t)$ and $E(t)$ at $\tilde{t}_{i}$ 's are specified by:

$$
\begin{array}{ll}
e\left(\tilde{t}_{2 i}\right)=\frac{1}{131,072}, \quad i=0, \ldots, 9, \quad e\left(\tilde{t}_{2 i+1}\right)=\frac{-1}{131,072}, \quad i=0, \ldots, 8, \\
E\left(\tilde{t}_{2 i}\right)=\frac{1}{262,144}, \quad i=0, \ldots, 9, \quad E\left(\tilde{t}_{2 i+1}\right)=\frac{-1}{262,144}, \quad i=0, \ldots, 8 .
\end{array}
$$

Therefore,

$$
\frac{-1}{131,072} \leq e(t) \leq \frac{1}{131,072}, \quad \frac{-1}{262,144} \leq E(t) \leq \frac{1}{262,144}, \quad t \in[0,1] .
$$

Proposition IV The errors of approximating the circular arc using the nonic Bézier curve in Theorem 1 at any $t \in[0,1]$ are given by

$$
\begin{aligned}
e(t)= & \frac{1}{131,072}-\frac{81 t}{16,384}+\frac{8721 t^{2}}{16,384}-\frac{2907 t^{3}}{128}+\frac{13,0815 t^{4}}{256}-\frac{223,839 t^{5}}{32}+\frac{2,028,117 t^{6}}{32} \\
& -401,166 t^{7}+\frac{3,677,355 t^{8}}{2}-6,249,100 t^{9}+15,984,540 t^{10}-31,000,320 t^{11} \\
& +45,601,920 t^{12}-50,512,896 t^{13}+41,425,920 t^{14}-24,379,392 t^{15} \\
& +9,732,096 t^{16}-2,359,296 t^{17}+262,144 t^{18}, \quad \forall t \in[0,1] .
\end{aligned}
$$

\section{Discussion}

In this section, the proofs of Theorem 1 and Propositions I, II, III, and IV besides other demonstrations are given.

Proof of Theorem 1 We begin by considering the polynomials $x(t)$ and $y(t)$ in Eq. (11) and substituting them into the error function $e(t)$ in (7). Disposition of the phrase and performing several simplifications give an equation that is simplified to a polynomial of degree eighteen.

The approximation conditions are satisfied if the error function is equalized with the polynomial of least deviation among all monic polynomials of degree eighteen. So, the last equation which exemplifies the error function has to be equalized with the Chebyshev polynomial of first kind of degree eighteen, $\tilde{T}_{18}(2 t-1) / 131,072$. We know that $\tilde{T}_{18}(u)=$ $\cos (18 \arccos (u)), u \in[-1,1]$ is the unique monic polynomial of degree eighteen that has the least deviation from the origin. Comparing the coefficients of equal powers of both sides and using the utilities of the computer algebra system in Mathematica, the solution that fulfills the conditions in (10) is established. Unfortunately, the solution is a collection of lengthy fractions and radicals, which makes it impractical to write down the values of the parameters in this paper, so we write them in decimal forms as in the theorem. This shows that $p$ fulfills the conditions of the approximation problem. The proof of the error formula for $E(t)$ is clear from the relation to $e(t)$. This completes the proof of Theorem 1 . 
Figure 3 Circular arc and its nonic Bézier curve in Theorem 1

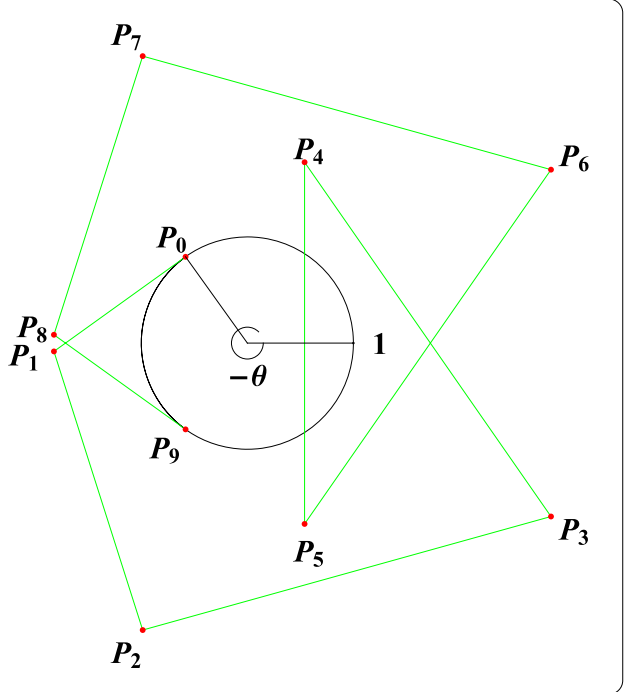

Figure 4 Euclidean Error of the nonic Bézier curve in Theorem 1

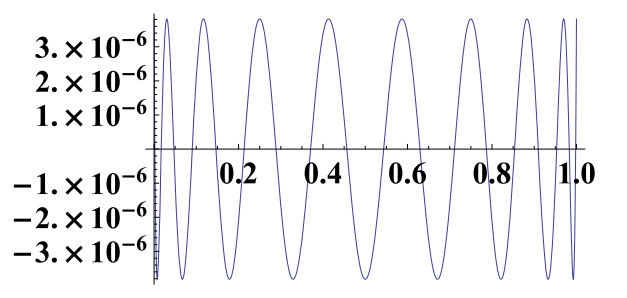

The circular arc and the approximating Bézier curve are plotted in Fig. 2 for $t \in\left[0, \frac{1}{2}\right]$. The circular arc and its nonic Bézier curve are plotted in Fig. 3. The resulting error between the curve and the approximation is not identified by the human eye which is clear from the figure of the corresponding error plotted in Fig. 4.

The resulting Bézier curve reveals a brilliant positioning of the Bézier points to embrace about $5 / 4$ of the whole circle whilst possessing the Chebyshev error. We could not foresee a nonic polynomial to approximate more than $5 / 4$ of full circle further accurately than this approximation.

Proof of Proposition I Immediate substitution of the values of $t_{i}$ in $e(t)$ gives $e\left(t_{i}\right)=0, i=$ $1,2, \ldots, 18$. Since $e(t)$ is a polynomial of degree 18 and has 18 roots, therefore, these ones are all the roots. The error function $E(t)$ has the same roots as $e(t)$ because $E(t)=0$ iff $\sqrt{x^{2}(t)+y^{2}(t)}=1$ iff $x^{2}(t)+y^{2}(t)=1$ iff $e(t)=0$.

The approximating nonic Bézier curve $p$ in Theorem 1 and the circular arc $c$ intersect at the points $p\left(t_{i}\right)=c\left(t_{i}\right), i=1,2, \ldots, 18$.

Proof of Proposition II The derivative of $e(t)$ is a polynomial of degree 17. Substituting the 17 parameters $\tilde{t}_{1}, \ldots, \tilde{t}_{17}$ into the derivative gives $e^{\prime}\left(\tilde{t}_{i}\right)=0, \forall i=1, \ldots, 17$. The polynomial $e^{\prime}(t)$ has degree 17; consequently, these are all internal critical points. Inspecting the end points adds $\tilde{t}_{0}=1, \tilde{t}_{18}=0$ to the critical points. For all $t \in[0,1]$, we have $1-\frac{1}{131,072} \leq$ $x^{2}(t)+y^{2}(t) \leq 1+\frac{1}{131,072}$, thence $\sqrt{x^{2}(t)+y^{2}(t)} \neq 0, \forall t \in[0,1]$. Differentiate $E(t)$ and counter 
equate to 0 to acquire $\frac{e^{\prime}(t)}{\sqrt{x^{2}(t)+y^{2}(t)}}=0$ iff $e^{\prime}(t)=0$. Therefore, $e(t)$ and $E(t)$ reach the extrema at the same values. This finishes the proof of the proposition.

The disagreement in the values of $E\left(\tilde{t}_{i}\right)$ for odd and even values of $i$ occurs because $e(t)$ equioscillates between $\pm \frac{1}{8192}$ and $\frac{1}{2^{17}(2-\epsilon)} \leq E(t) \leq \frac{1}{2^{17}(2+\epsilon)}$, where $\epsilon=\max _{0 \leq t \leq 1}|E(t)|$.

Proof of Proposition III The values of the error functions $e(t)$ and $E(t)$ at the parameters $\tilde{t}_{i}$ are specified by:

$$
\begin{array}{ll}
e\left(\tilde{t}_{2 i}\right)=\frac{1}{131072}, \quad i=0, \ldots, 9, \quad e\left(\tilde{t}_{2 i+1}\right)=\frac{-1}{131072}, \quad i=0, \ldots, 8 . \\
E\left(\tilde{t}_{2 i}\right)=\frac{1}{262144}, \quad i=0, \ldots, 9, \quad E\left(\tilde{t}_{2 i+1}\right)=\frac{-1}{262144}, \quad i=0, \ldots, 8 .
\end{array}
$$

Therefore,

$$
\frac{-1}{131072} \leq e(t) \leq \frac{1}{131072}, \quad \frac{-1}{262144} \leq E(t) \leq \frac{1}{262144}, \quad t \in[0,1]
$$

Substituting the parameters in the error functions confer to the parities. The specifics of the proof of the proposition are left to the reader.

Proof of Proposition IV This is a forthright conclusion of Theorem 1. The specifics of the proof of the proposition are left to the reader.

Employ the relation between $E(t)$ and $e(t)$ to obtain:

$$
\begin{aligned}
E(t) \cong & \frac{1}{262,144}-\frac{81 t}{32,768}+\frac{8721 t^{2}}{32,768}-\frac{2907 t^{3}}{256}+\frac{130,815 t^{4}}{512}-\frac{223,839 t^{5}}{64}+\frac{2,028,117 t^{6}}{64} \\
& -200,583 t^{7}+\frac{3,677,355 t^{8}}{4}-3,124,550 t^{9}+7,992,270 t^{10}-15,500,160 t^{11} \\
& +22,800,960 t^{12}-25,256,448 t^{13}+20,712,960 t^{14}-12,189,696 t^{15} \\
& +4,866,048 t^{16}-1,179,648 t^{17}+131,072 t^{18}, \quad \forall t \in[0,1] .
\end{aligned}
$$

\section{Conclusion}

The polynomial of best uniform approximation of degree nine is given for a circular arc that approximates with order eighteen rather than ten. The error function equioscillates 19 times rather than 11 times that are guaranteed by the theorems of Chebyshev and Borel without a method to find it. The error function is studied and characterized, and its extrema and zeros are derived.

Acknowledgements

The author owes thanks to the reviewers for helpful and invaluable comments and suggestions for improving an earlier version of this paper.

Funding

This paper was funded by Jordan University of Science and Technology. 
Competing interests

The author declares that there are no competing interests in this paper.

\section{Authors' contributions}

The author declares that no other authors have contributed to the manuscript. All authors read and approved the final manuscript.

\section{Publisher's Note}

Springer Nature remains neutral with regard to jurisdictional claims in published maps and institutional affiliations.

Received: 4 March 2019 Accepted: 17 May 2019 Published online: 29 May 2019

\section{References}

1. Ahn, Y.J., Kim, Y.S., Shin, Y.: Approximation of circular arcs and offset curves by Bézier curves of high degree. J. Comput. Appl. Math. 167(2), 405-416 (2004)

2. Bézier, P.: The Mathematical Basis of the UNISURF CAD System. Butterworth-Heinemann, Newton (1986). ISBN $0-408-22175-5$

3. Blinn, J.: How many ways can you draw a circle? IEEE Comput. Graph. Appl. 7(8), 39-44 (1987)

4. Dokken, T., Dæhlen, M., Lyche, T., Mørken, K.: Good approximation of circles by curvature-continuous Bézier curves. Comput. Aided Geom. Des. 7, 33-41 (1990)

5. Farin, G.: Curves and Surfaces for Computer Aided Geometric Design. Academic Press, Boston (1988)

6. Goldapp, M.: Approximation of circular arcs by cubic polynomials. Comput. Aided Geom. Des. 8, 227-238 (1991)

7. Höllig, K., Hörner, J.: Approximation and Modeling with B-Splines, Titles in Applied Mathematics, vol. 132, SIAM, Philadelphia (2013)

8. Kim, S.H., Ahn, Y.J.: An approximation of circular arcs by quartic Bézier curves. Comput. Aided Des. 39(6), 490-493 (2007)

9. Natanson, I.P.: Constructive Function Theory, Vol. 1, Uniform Approximation. Ungar, New York (1964)

10. Rababah, A.: Taylor theorem for planar curves. Proc. Am. Math. Soc. 119(3), 803-810 (1993)

11. Rababah, A.: High accuracy Hermite approximation for space curves in $\mathfrak{\Re}^{d}$. J. Math. Anal. Appl. 325(2), $920-931$ (2007)

12. Rababah, A.: The best uniform quadratic approximation of circular arcs with high accuracy. Open Math. 14, 118-127 (2016)

13. Rababah, A.: Quartic approximation of circular arcs using equioscillating error function. Int. J. Adv. Comput. Sci. Appl. 7(7), 590-595 (2016)

14. Rababah, A.: The best uniform cubic approximation of circular arcs with high accuracy. Commun. Math. Appl. 7(1), $37-46$ (2016)

\section{Submit your manuscript to a SpringerOpen ${ }^{\odot}$ journal and benefit from:}

- Convenient online submission

- Rigorous peer review

- Open access: articles freely available online

- High visibility within the field

- Retaining the copyright to your article 